\title{
BMJ Open Safety and effectiveness of a Tai Chi- based cardiac rehabilitation programme for chronic coronary syndrom patients: study protocol for a randomised controlled trial
}

\author{
Jing Ma, ${ }^{1}$ Jian wei Zhang (D) , ${ }^{2}$ Hua Li, ${ }^{3}$ Lian shan Zhao, ${ }^{4}$ Ai ying Guo, ${ }^{3}$ \\ Zai hao Chen, ${ }^{5}$ Wen Yuan, ${ }^{5}$ Tian ming Gao, ${ }^{2}$ Ya meng $\mathrm{Li}^{2}{ }^{2}$ Cui han $\mathrm{Li}^{5}{ }^{5}$ \\ Hong wei Wang, ${ }^{2}$ Bo Song, ${ }^{2}$ Yu long Lu, ${ }^{2}$ Mei ze Cui, ${ }^{2}$ Qiu yang Wei, ${ }^{2}$ \\ Shao jun Lyu, ${ }^{2}$ Heng chan Yin ${ }^{2}$
}

To cite: Ma J, Zhang Jwei, $\mathrm{Li} \mathrm{H}$, et al. Safety and effectiveness of a Tai Chibased cardiac rehabilitation programme for chronic coronary syndrom patients: study protocol for a randomised controlled trial. BMJ Open 2020;10:e036061. doi:10.1136/ bmjopen-2019-036061

- Prepublication history and additional material for this paper are available online. To view these files, please visit the journal online (http://dx.doi. org/10.1136/bmjopen-2019036061).

JM and JwZ contributed equally.

JM and JWZ are joint first authors.

JM and JwZ are joint senior authors.

Received 12 December 2019 Revised 16 March 2020 Accepted 20 May 2020

Check for updates

(C) Author(s) (or their employer(s)) 2020. Re-use permitted under CC BY-NC. No commercial re-use. See rights and permissions. Published by BMJ.

For numbered affiliations see end of article.

\section{Correspondence to} Professor Shao jun Lyu; 113121860699@163.com; shaojunl@hotmail.com and Professor Heng chan Yin; yinhengchan@bnu.edu.cn

\section{ABSTRACT}

Introduction Preliminary evidence from clinical observations suggests that Tai Chi exercise may offer potential benefits for patients with chronic coronary syndrom (CCS). However, the advantages for CCS patients to practice Tai Chi exercise as rehabilitation have not been rigorously tested and there is a lack of consensus on its benefits. This study aims to develop an innovative Tai Chi Cardiac Rehabilitation Program (TCCRP) for CCS patients and to assess the efficacy, safety and acceptability of the programme.

Methods and analysis We propose to conduct a multicentre randomised controlled clinical trial comprising of 150 participants with CCS. The patients will be randomly assigned in a 1:1 ratio into two groups. The intervention group will participate in a supervised TCCRP held three times a week for 3 months. The control group will receive supervised conventional exercise rehabilitation held three times a week for 3 months. The primary and secondary outcomes will be assessed at baseline, 1 month, 3 months after intervention and after an additional 3-month follow-up period. Primary outcome measures will include a score of 36-Item Short Form Survey and Chinese Perceived Stress Scale. The secondary outcome measures will include body composition, cardiopulmonary exercise test, respiratory muscle function, locomotor skills, echocardiogram, New York Heart Association classification, heart rate recovery time and laboratory examination. Other measures also include Seattle Angina Scale, Pittsburgh Sleep Quality Index, Patient Health Questionnaire-9, Generalized Anxiety Disorder-7 and Berg Balance Scale. All adverse events will be recorded and analysed.

Ethics and dissemination This study conforms to the principles of the Declaration of Helsinki and relevant ethical guidelines. Ethical approval has been obtained from the Ethics Committee of Chinese People's Libration Army General Hospital (approval number: S2019-060-02). Findings from this study will be published and presented at conferences for widespread dissemination of the results.

Trial registration number NCT03936504.

\section{Strengths and limitations of this study}

- The proposed research study is unique and the first study about a Bafa Wubu of Tai Chi, which is a new Tai Chi school.

- Tai Chi Cardiac Rehabilitation Program in this study was specifically designed for patients with chronic coronary syndrom.

- This is the first time for Tai Chi study to develop a comparative training system to match the conventional exercise.

- It is difficult to exclude the effect of other physical activities due to difficulty of monitoring.

- The blinding of participants is unachievable in this trial; however, efforts will be made to ensure that the data are blinded.

\section{INTRODUCTION}

Coronary heart disease (CHD) remains the major cause of morbidity and mortality worldwide. CHD responsible for about one in every seven deaths; and, China Heart Society is predicted to continue until 2030, accounting for $14 \%$ of all deaths globally. ${ }^{12}$ Despite the use of effective Western medicine treatments, the incidence of CHD continues to rise and is associated with a high mortality rate. Cardiac rehabilitation (CR), especially exercise training, is proven to significantly alleviate the cardiac symptoms, preserve the heart function and improve the clinical outcomes. Therefore, CR is recommended by many guidelines of different countries. ${ }^{3-8}$ However, latest study demonstrated only $24.4 \%$ CR-eligible medicare beneficiaries participated in CR and marked disparities were observed. ${ }^{9}$ Besides limitation of medical resource, poor reports of patients remain main reason. An 
effective and attractive CR training system is urgently needed.

These years, the integration of traditional Chinese medicine (TCM) with Western medicine to treat CHD has made great progress. As an effective complementary therapy, TCM has been demonstrated to improve the prognosis of CHD patients. ${ }^{10}$ Tai Chi is an important element of TCM which combines the meridians and collateral theory, Yin-Yang theory and five-element theory. Tai Chi exercise contains three core elements, namely 'body', 'breath' and 'mind', as pronounced in Chinese as 'Xing', 'Qi' and 'Yi', respectively. The spirits of Tai Chi are summarised to 'building the body', 'conveying the breath' and 'using the mind'. Previous studies have shown that regular Tai Chi exercise was beneficial in improving psychological and physiological outcomes among CHD patients. ${ }^{11-14}$ Study by professor Salmorirago-Blocthcer has shown patients receiving Tai Chi exercise exhibited much better outcome than those receiving conventional exercise. $^{11}$

There are many schools in Tai Chi such as the Yangstyle, Wu-style, Chen-style, Wu-style and Sun-style, wherein each style takes a different approach in terms of the movements and forms. Furthermore, as Tai Chi exercise comprises many assorted movements that can also becomplex to perform, it is difficult to popularise and simplify the exercise, especially in elderly patients and patients with chronic diseases.

Based on the prior insights into the Tai Chi movements obtained from our studies and other work, ${ }^{1516}$ our research team developed an innovative Tai Chi Cardiac Rehabilitation Program (TCCRP) specifically for CHD patients. However, as the value of TCCRP has not yet to be clinically proven, a clinical trial is required to validate the benefits of adopting this exercise for CHD patients.

CHD can be categorised as either acute coronary syndrome or chronic coronary syndrome (CCS) due to pathophysiological features and clinical prognosis because it is dynamic process of atherosclerotic plaque accumulation and functional alterations of coronary circulation. ${ }^{17}$ Between the two process, most CHD patients stay in the CCS state. Latest studies revealed patients with CCS benefit a lot from CR programmes characterised by life style therapy. ${ }^{17}$ So we chose CCS patients as our subjects. This study aims to assess the efficacy, safety and acceptability of TCCRP for CCS patients.

The primary hypothesis is that TCCRP (the intervention group) will improve the life quality and reduce the stress when compared against conventional exercise rehabilitation (CER, the control group). Secondary objectives are to evaluate the effects of TCCRP on body composition, cardiopulmonary exercise test, respiratory muscle function, locomotor skills, echocardiogram, New York Heart Association (NYHA) classification, heart rate recovery time and laboratory examination. Other indicators measured will include Seattle Angina Scale (SAQ), Pittsburgh Sleep Quality Index (PSQI), Patient Health Questionnaire-9 (PHQ-9), Generalised Anxiety

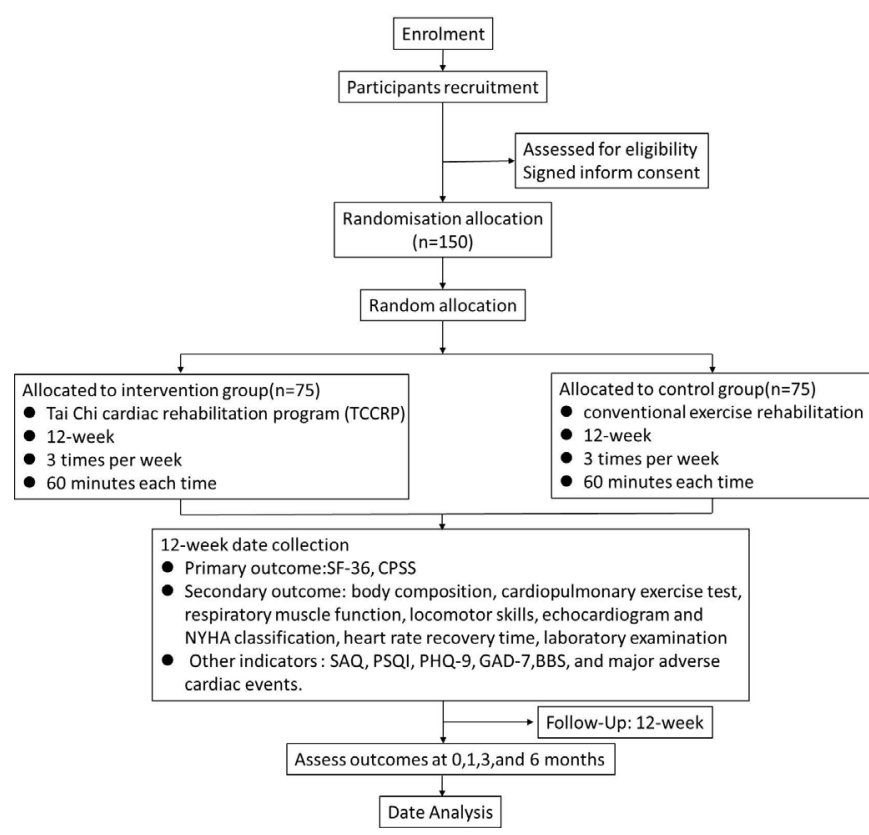

Figure 1 Flow diagram of study design. BBS, Berg Balance Scale; CPSS, Chinese Perceived Stress Scale;GAD-7, Generalized Anxiety Disorder-7; NYHA, New York Heart Association; PSQI, Pittsburgh Sleep Quality Index; PHQ-9, Patient Health Questionnaire-9;SF-36, SAQ, Seattle Angina Scale; SF-36 Health Survey.

Disorder-7 (GAD-7), Berg Balance Scale (BBS) and major adverse cardiac events. Additional objectives to be explored will include (1) the influence of potential factors on the adherence to the TCCRP, (2) the safety of the TCCRP and (3) individual experiences and acceptability following the TCCRP.

\section{METHODS/DESIGN \\ Study design}

This is a prospective, multicentre, randomised controlled clinical trial comparing a TCCRP with CER with an allocation ratio of $1: 1$. The study period lasts for 6 months including a 3-month supervised intervention and a 3-month follow-up with the primary outcomes measured at baseline, 1 month, 3 months and 6 months. Secondary outcomes will be measured at baseline and after a 3-month intervention period. A brief flowchart of the entire study is shown in figure 1 and the schedule of events is provided in table 1. The study protocol is reported according to Standard Protocol Items: Recommendations for Intervention Trials 2013 (SPIRIT). A SPIRIT checklist is provided in the online supplementary additional file 1 . The study protocol was submitted to the Ethics Committee of Chinese PLA General Hospital on 26 January 2019. After two revisions, final version 3 of the protocol was approved on 2 June 2019 (approval number: S2019-060-02).

\section{Sample size calculation}

Sample size calculation will be based on the coprimary outcomes of the randomised controlled trial. The SF-36 Health Survey (SF-36) and Chinese Perceived Stress Scale 
Table 1 Schedule for data collection; outcome measures per visits

\begin{tabular}{llllll}
\hline Items & $\begin{array}{l}\text { Phase I: } \\
\text { screening }\end{array}$ & $\begin{array}{l}\text { Phase II: } \\
\text { baseline }\end{array}$ & $\begin{array}{l}\text { Phase III: } \\
\text { 1 month }\end{array}$ & $\begin{array}{l}\text { Phase IV: } \\
\mathbf{3} \text { months }\end{array}$ & $\begin{array}{c}\text { Phase V: } \\
\mathbf{6} \text { months }\end{array}$ \\
\hline Inclusion/exclusion criteria & $\sqrt{ }$ & & & & \\
Diagnostic index & $\sqrt{ }$ & & & \\
Signed informed consent & $\sqrt{ }$ & & & \\
Randomisation and allocation & $\sqrt{ }$ & & & $\sqrt{ }$ \\
Safety index & $\sqrt{ }$ & $\sqrt{ }$ & $\sqrt{ }$ & \\
General clinical information & & $\sqrt{ }$ & & $\sqrt{ }$ & \\
Primary outcomes & & $\sqrt{ }$ & & $\sqrt{ }$ & \\
Secondary outcomes & $\sqrt{ }$ & & $\sqrt{ }$ & $\sqrt{ }$ \\
Other indicators & & $\sqrt{ }$ & & $\sqrt{ }$ \\
Recurrent cardiovascular events & & $\sqrt{ }$ & $\sqrt{ }$ \\
Adherence & & & $\sqrt{ }$ & $\sqrt{ }$ \\
Adverse events & & & & \\
Summary at the end of the study & & & & \\
\hline
\end{tabular}

(CPSS) are being set as the coprimary outcome and used for sample size calculation. Sample size was calculated on the basis of the changes in the SF-36 and CPSS between comparison groups with a significance level of $5 \%$ and a two-tailed critical region to ensure the same effect size with $80 \%$ power by G*Power V.3.1.9.4 software. The means and their SDs (mean \pm SD) of the SF-36 and CPSS in the control and intervention group were $(64.30 \pm 13.11$, $71.79 \pm 16.03)^{18}$ and $(42.31 \pm 8.17,35.15 \pm 6.82),{ }^{19}$ respectively, at postintervention according to the published literature. Because the sample size calculation of CPSS was less than SF-36, the sample size calculation of SF-36 was selected. This would require 122 participants, inflated to about 150 to account for the loss to follow-up of approximately $20 \%$ of participants, with 75 participants being assigned to each group.

\section{Participants}

Inclusion criteria

1. Man or non-pregnant women aged from 18 to 80 years.

2. Patients who met the diagnosis criteria of chronic coronary syndrome included in Knuuti et al. ${ }^{17}$

3. NYHA class I and II.

4. Participants who understood the purpose of the clinical trial and voluntarily participate with signed informed consent.

\section{Exclusion criteria}

1. Acute myocardial infarction within 2 weeks.

2. Severe aortic stenosis.

3. Hypertrophic cardiomyopathy.

4. Severe valvular heart disease.

5. Malignant tachyarrhythmia.

6. Poor patient report and incompletion of the clinical trial for not satisfying the requirements.
7. Patients with abnormal motor function caused by nervous system deterioration, motor system disease or rheumatic disease.

8. Those who regularly practice Tai Chi during the previous 3 months.

\section{Setting and recruitment}

This multicentre study will be performed at the Beijing Normal University in China. Recruitment and exercise training will occur at the Chinese PLA General Hospital, China, Beijing Shuili Hospital, China and Anzhen Community Health Service Center, Beijing Chaoyang District, China. One hundred and fifty participants are to be recruited and the recruitment is scheduled to begin in October 2019. Combinations of advertising strategies include flyers within the hospital, advertisements in the print, online media, a major messaging platform (WeChat), clinics and databases.

\section{Randomisation, allocation concealment and blinding}

After informed consent is signed, all patients will be randomised into either an intervention group receiving a 12-week TCCRP or a control group receiving CER. The random allocation sequence will be produced by an independent statistician via the PLAN sentences of the statistical software SAS V.9.2 in a 1:1 ratio. Next, these assignments will be sent to a study staff member, exclusive to the study coordinator or principal investigator, who will store them into sealed, opaque envelopes with date and signature labels placed over the seals of the envelopes. The randomisation envelopes will not be opened unless a participant meets eligibility criteria, completes the informed consent and undergoes a baseline assessment. Informed consent will be obtained by two blinded research assistants prior to the baseline assessment. Eligible participants will receive the 
information about this trial and have an informed discussion with two trained research assistants regarding the information provided. The study is conducted in three different cycles. Each cycle consists of a TCCRP group (intervention group) and CER group (control group). Each resulting group consists of 25 patients, equating to 50 patients participating in each cycle, with a total of 150 patients over the course of the three cycles comprising the study. The instructors are randomly assigned to the three cycles.

Given the nature of the intervention, it is impossible to blind the patients or any personnel who are directly involved in conducting the programmes. However, all outcome assessors, laboratory technicians, data managers and statisticians will be kept blind of the treatment allocations.

\section{Patient and public involvement}

We gave priority to patients as far as possible. Patients are the major factor to be consider when designing the trial, subject recruitment and data sharing. Since the very beginning of our study, we have constructed a patient public involvement group. During the group meeting, patient public involvement group members were informed about how the study will be conducted. We always take a lot of time to communicate with patients about how to improve the detail of study to improve their health and they have given some good advice. For example, the patients suggested us to prepare simple booklet to introduce our study during the outpatients' visit and by internet, also to enlarge the recruitment extent by WeChat and app of our hospital. We planned to disseminate our research to the participants and the public, such as publicising our research in hospital official accounts and various academic lectures.

\section{Interventions}

\section{TCCRP group}

Patients in the intervention group will receive TCCRP conducted by a CR team consisting of cardiologists, cardiology nurses, Tai Chi coaches and research assistants. Procedures and activities of the TCCRP include (1) Tai Chi exercise, (2) evaluation of exercise ability, (3) education covering topics related to the exercise and (4) a series of adherence strategies.

\section{TCCRP prephase: a 2-week exercise before the start of exercise}

\section{TCCRP training}

Six professional coaches with at least 10 years of Tai Chi teaching experience will be employed to teach and guide the participants' training. TCCRP will include (a) traditional Tai Chi warm-up exercises, followed by (b) Bafa Wubu of Tai Chi, (c) Tai Chi elastic belt exercise and (d) Tai Chi cool-down exercises.

a. Tai Chi warm-up exercises $(10 \mathrm{~min})$ will include traditional breathing methods (full-body breathing), weight shifting, arm-swinging and so on. These exercises will help release tension in the physical body, incorporate mindfulness and imagery into movement, increase breathing awareness and promote overall relaxation of the body and mind.

b. The core Tai Chi movements $(30 \mathrm{~min})$ will be adapted from the Bafa Wubu of Tai Chi (also known as 'eight methods and five footwork', figure 2), which include introductory routines to Tai Chi characterised with simple structures and rich connotations performed repetitively. Technically speaking, the 'Bafa' consists eight hand techniques, namely, 'Peng (warding off), Lu (rolling back), Ji (pressing), An (pushing), Cai (pulling down), Lie (splitting), Zhou (elbowing) and

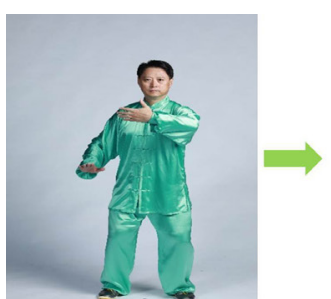

Peng (warding off)

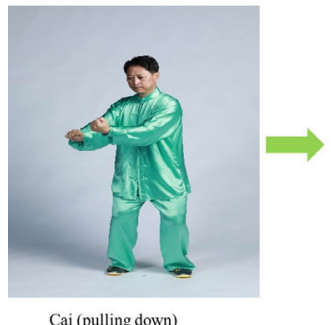

Cai (pulling down)

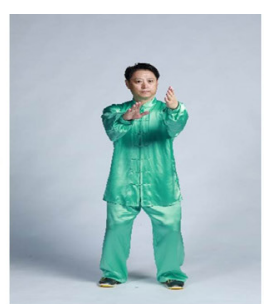

Lu (rolling back)

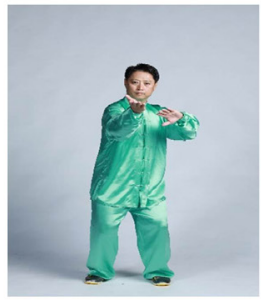

Lie (splitting)

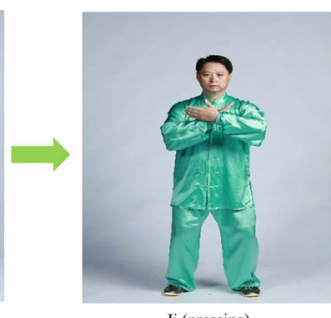

Ji (pressing)

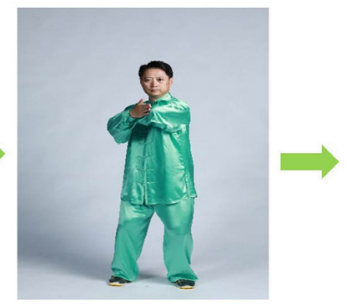

Zhou (elbowing)

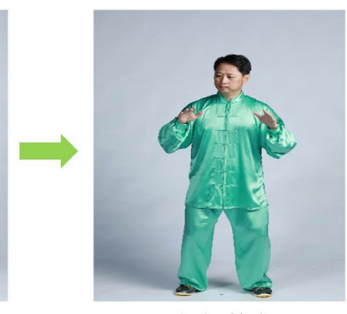

An (pushing)

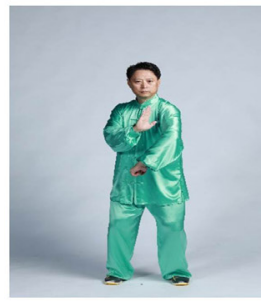

Kao (shouldering)

Figure 2 Exemplary Bafa Wubu of Tai Chi; the pictures have taken the portrait right; the fugleman is the developer of TCCRP. The 'Bafa' consisted of eight hand techniques are shown in figure 2. Each figure shows each hand technique, namely, 'Peng (warding off), Lu (rolling back), Ji (pressing), An (pushing), Cai (pulling down), Lie (splitting), Zhou (elbowing) and Kao (shouldering)'. 
Kao (shouldering)'; while the 'Wubu' consists five footwork, namely, 'Jin (advancement), Tui (retreat), Gu (shifting left), Pan (shifting right) and Ding (central equilibrium).

c. Tai Chi combined with lightweight resistance band exercises (10 min) will include Tai Chi 'Open and Close' movement, Tai Chi Spinning movement and Tai Chi Twining movement.

d. Tai Chi cool-down exercises $(10 \mathrm{~min})$ will include various relaxation methods such as regulating breath, regulating body and regulating mind.

Patients are required to practice the TCCRP until they master it. Mastery will be determined by the professional coaches. The mastery of Tai Chi was assessed and quantified due to the following six factors: the body shape should keep straight and upright; the gravity centre shift is right; the action moves in an order; moving speed has a sense of rhythm; action and movement contain wring and screwing and every set consumes similar time. We have constructed a testing committee including 10 Tai Chi experts who had discussed together and summarised a scoring criteria concerning the above six dimensions. Six professional coaches with more than 10 years of Tai Chi teaching experiences were trained about the scoring system and then coached the subjects. At the end of learning stage, three coaches were chosen randomly to work as the examiner. The professional coaches scored on a percentile basis according to the above six dimensions. Only the participants who gained an average score higher than 80 would be certificated to be qualified and move on to the trial step.

2. Evaluation of exercise ability (week-1 to 0 ):

a. Evaluation is conducted by cardiologists and physiotherapists.

b. Evaluation is based on reviewing medical history, cardiopulmonary exercise test results and the performance of Tai Chi (heart rate and oxygen consumption will be recorded while performing Tai Chi).

c. Evaluation results will guide the goal-setting process during consultation.

3. Education covering topics related to exercise:

a. Basic knowledge of chronic heart disease.

b. Basic knowledge of exercise-based CR.

\section{TCCRP exercise phase: a 12-week intervention period}

Participants will perform the TCCRP three times a week for 12 weeks. Each training session is $60 \mathrm{~min}$ and includes Tai Chi warm-up exercises (10 min), Bafa Wubu of Tai Chi (30 min), Tai Chi combined with lightweight resistance band exercises (10 min) and Tai Chi cool-down exercises (10 min). All participants will be encouraged to practice Tai Chi according to an instructional video until the end of 12-week period.

Researchers will record the subjects' heart rate and blood pressure before and after training. During the training, the exercise intensity will be assessed by the Borg Rating of Perceived Exertion Scale, ${ }^{20}$ which is a frequently used quantitative measure of perceived exertion during exercise.

\section{TCCRP follow-up phase: a 12-week follow-up period}

After the 12-week TCCRP intervention, there will be a 12-week follow-up period that excludes any active rehabilitation. During the follow-up period, the participants will be asked to fill out forms to record the times and durations of their Tai Chi exercise or other physical activities and any incidence of a major adverse cardiac event. The forms will be returned to the researchers for follow-up each week by email or WeChat.

\section{CER group (control group)}

Participants in the control group will receive a CER three times a week for 12 weeks. Each training session lasts for $60 \mathrm{~min}$, including ordinary warm-up exercises $(10 \mathrm{~min})$, aerobic activity $(30 \mathrm{~min})$, resistive exercise $(10 \mathrm{~min})$ and cool-down exercises $(10 \mathrm{~min})$. Each training session includes (1) an active warm-up including arm-swinging, gentle stretches of the neck, shoulders, spine, arms and legs, (2) an aerobic activity comprising primarily cycle ergometer exercise, (3) resistive exercise mainly including resistance band exercises and (4) a cool-down session involving active and static stretching exercises with primary body movements.

The CER programme is consistent with the current recommended guidelines of moderate intensity exercises (50\%-80\% heart rate reserve; rated perceived exertion 11-13) for CHD patients. Our programme is individually tailored to each participant alongside close supervision. The programme will be introduced and increased in duration and intensity gradually to achieve the target of moderate-intensity exercise.

\section{Concomitant treatment}

Participants in both groups will continue routine medications such as aspirin, metoprolol, antiplatelet and anticoagulant drugs or beta-adrenergic blockers, according to patients' respective conditions and will maintain their usual treatment visits throughout the study. All procedures and medication prescriptions will be determined by physicians following the clinical guidelines. ${ }^{21} 22$ The specific date and reasons of any medical therapy changes will be recorded in the case report form (CRF).

\section{Outcome measures}

All outcome measures will be collected by three research assistants at 2 weeks (baseline), 1 month, 3 months (at the end of intervention) and 6 months (at the 3-month follow-up). Demographic information collected will include age, gender, ethnicity, marital status, education level, accommodation type and postal address. Clinical information will also be obtained from the patients' clinical records by a member of their hospital research team. All data collected from the assistants and therapists will be stored in a dedicated computer for the study and will be kept in a secure and lock-protected location. 


\section{Primary outcome measures}

1. The SF-36 is a multipurpose, short-form health survey with only 36 questions. ${ }^{23}$ SF-36 items cover eight domains: physical functioning, role limitations due to physical health problems, body pain, general health, vitality, social functioning, role limitations due to emotional problems and mental health. Higher scores indicate higher levels of health. SF-36 will be evaluated at baseline, 1 month, 3 months (at the end of intervention) and 6 months (at the 3-month follow-up).

2. CPSS is a self-rated questionnaire that assesses perceived stress. ${ }^{24}$ CPSS consists 14 items that are divided into two categories: sense of tension and loss of control. Higher scores indicate higher levels of stress. CPSS will be evaluated at baseline, 1 month, 3 months (at the end of intervention) and 6 months (at the 3-month follow-up).

\section{Secondary outcome measures}

1. Body composition measurements will include fat mass, body fat (percentage), fat-free mass and lean body mass. These measurements will be taken by bioelectrical impedance analysis using an Inbody 770 (Biospace Co) at baseline, 1 month, 3 months (at the end of intervention) and 6 months (at the 3-month follow-up).

2. Cardiopulmonary exercise test (CPET) is an objective method being increasingly used in a wide spectrum of clinical practice for assessing the functional capacity of CHD patients. Indexes include changes in $\mathrm{VO}_{2}$ peak and VAT and $\mathrm{VE} / \mathrm{VCO}_{2}$ slope in the CPET at the end of the 3-month intervention.

3. Respiratory muscle function will be measured by POWER breath K-5. Indexes include changes in SIndex, peak of inspiratory velocity and power at the end of the 3-month intervention.

4. Locomotor skills include handgrip strength, balance and flexibility. Handgrip strength, which is used to determine the maximum isometric strength of the hand and forearm muscles, will be measured using the handgrip strength dynamometer produced by CAMRY (product type EH101). The best result from repeated tests of each hand will be recorded. The balance will be evaluated by using the time duration until losing balance. We respectively investigated the time duration of standing on one foot with eyes closed, standing on one foot with eyes open, strengthening Romberg' s test. ${ }^{25}$ The participants were tested three times to record their time until they lost their balance. Finally, the best of the three was selected. Time difference was calculated as the time duration after treatment minus that before treatment. Flexibility will be measured by seated forward flexion test. Locomotor skills will be evaluated at baseline and at the end of the 3-month intervention.

5. An echocardiogram comprising left ventricular end diastolic (LVED), volume index (Vi) and (left ventricular ejection fraction) LVEF using echocardiography will be assessed at baseline and at the end of the 3-month intervention. NYHA classification will also be evaluated at baseline and at the end of the 3-month intervention.

6. Heart rate recovery time will be measured. Heart rate recovery time will record heart rate $1-6$ min after Tai Chi exercise, power cycling and resistance exercise.

7. A laboratory examination will be performed that includes glycolipid metabolism, inflammatory factor level, immunologic function and oxidative stress index. The laboratory examination will be evaluated at baseline and at the end of the 3-month intervention.

8. The BBS is a widely used clinical test of a person's static and dynamic balance abilities ${ }^{26}$ and comprises of a set of 14 simple balance related tasks, ranging from standing up from a sitting position to standing on one foot. Total score ranges from 0 to 56 , with 0 to 20 corresponding to a high fall risk, 21 to 40 a medium fall risk and 41 to 56 a low fall risk. The BBS will be evaluated at baseline and at the end of the 3-month intervention.

9. The PSQI is a self-rated questionnaire that assesses sleep quality and disturbances. ${ }^{27}$ It contains 19 self-answered questions for the subject and 5 peeranswered questions for the bed partner or a roommate (if one is available). The scores from seven categories are added to calculate the index, ranging from 0 to 21. A score of 0 indicates no disturbance in sleep or good sleep quality, whereas higher scores indicate poorer sleep quality. The PSQI will be evaluated at baseline and at the end of the 3-month intervention.

10. PHQ-9 and GAD-7 are validated self-answered questionnaires that assess levels of depression and anxiety. Higher scores reflect greater levels of anxiety and depression. PHQ-9 and GAD-7 will be evaluated at baseline and at the end of the 3-month intervention.

11. The SAQ will be used to determine the total number of nine problems and the five aspects of coronary artery disease (CAD), including the degree of physical activity, the stability and frequency of angina, the degree of satisfaction of the treatment and the perception of the disease. The higher the score, the better the quality of life and body function. SAQ will be measured at baseline and at the end of the 3-month intervention.

12. A record will be made of any side effects and possible adverse reactions arising from the intervention.

\section{Safety measurements}

All study participants are monitored weekly during the study intervention for the occurrence of adverse events defined by any undesirable experience. All adverse events that occur during the study will be recorded on the adverse event CRF and will be evaluated for relevance to the intervention. All adverse events will also be reported to the Human Research Committee promptly in accordance with guidelines. 
Only patients who are eligible and capable of completing the test will undergo a CPET. For enrolled patients meeting the rehabilitation training standards, they will be stratified according to the degree of motion risk and appropriate exercise intensity and time will be adjusted based on their risk stratification. Before the CR exercise, researchers will educate patients about the CHD rehabilitation exercises, including training contraindications and exercise advisories regarding the respective CER and TCCRP exercises. Moreover, the CR Center of Chinese PLA General Hospital, Beijing Shuili Hospital and Anzhen Community Health Service Center are equipped with a comprehensive set of rescue equipment. A thorough contingency plan and rescue procedure for cardiovascular events have also been formulated prior to the commencement of the research. Should an adverse event occur during the exercise, the researchers will immediately initiate the contingency plan to circumvent the occurrence of any fatal outcomes.

\section{Data management and monitoring}

Beijing Normal University will be responsible for managing the data and performing statistical analyses. The research assistants will be responsible for checking the integrity of the completed CRF and for timely entry of the collected data into the EpiData Manager, a free data management software. The project manager will be responsible for initial data cleaning, identifying, coding and converting the data into the proper format for analysis. All investigators involved in data management and analysis will be blinded to treatment allocation. Regular monitoring by the sponsor of China National Center for Biotechnology Development will be performed according to ICH GCP. China National Center for Biotechnology Development will be responsible for monitoring the research progress and meet every 3 months. It will oversee all aspects of the trial delivery including protocol amendments, recruitment of participants, monitoring intervention fidelity, management of timelines and milestones, publication and dissemination plans. Each amendment of the protocol conforms to the GCP principles and is submitted to the ethics committee for approval.

\section{Statistical analysis}

Continuous variables will be described as mean \pm SD for normal distributions or median for non-normal distributions, categorical variables will be described as frequency. Baseline data mainly describe the clinical characteristic and features of the subjects. We also tested the equalisation of the two groups of variables. Continuous variables will be described as a two-sample Student's t-test for normal distributions or Wilcoxon test for non-normal distributions. Categorical variables will be described as the chi-square test. The group difference between intervention and control group at each time point (4 and 12 weeks after intervention or 12-week follow-up period) will be analysed using Student's t-test or Mann-Whitney U test. A two-way analysis of variance with repeated measures will be used to determine the effects of time and group on our dependent variables. A Bonferroni-adjusted post hoc analysis will be conducted when time-group interaction was detected. The analysis of primary or secondary outcomes will be based on an intention to treat principle, and participants who either drop out from the study or fail to adhere to the protocol will have their last known data carried forward. The missing data will be imputed using a multiple imputation method. All data will be analysed with SPSS V.21.0 (IBM) software packages. Statistical significance is defined as a two-sided $\mathrm{p}$ value $<0.05$.

\section{Adherence}

To motivate participants' adherence, the research group will use several strategies (eg, ancillary and post-trial care) to ensure the participants stays for the entire study period. (1) The participants will receive $36 \mathrm{CR}$ treatments free of charge, for a total price of about 10,000 RMB, (2) the participants will be entitled specialist outpatient priority plus and (3) participants will be given three free face-to-face health education lectures by specialists; In addition, participant who complete the protocol successfully will be rewarded with Wushu training clothing no matter which group they belongs to. During the 3-month treatment period, participants will be asked to practice strictly according to the training programme and will not be allowed to take part in any new or additional exercise programmes. Throughout the 3-month intervention period, the researchers will track the number of missed sessions for each participant during the intervention period. Participants' attendance will be monitored during each in-person session by staff-completed attendance forms and class sign-in sheets. The percentage of compliance will be documented on the CRF. The rate of patient compliance $=($ total planned number of times - number of absence)/total number of times $\times 100 \%$. A compliance rate of $80 \%$ or greater will be considered as good, whereas a compliance rate of less than $80 \%$ is considered as poor. An attendance of less than $20 \%$ will be considered as a dropout from the study.

\section{Ethics and dissemination}

This study conforms to the principles of the Declaration of Helsinki and relevant ethical guidelines. Ethical approval and informed consent form have been obtained from the Ethics Committee of Chinese PLA General Hospital (approval number: S2019-060-02). The study background and main objective as well as potential benefits and risks will be fully explained to the participants and their families. Findings from this study will be published and presented at conferences for widespread dissemination of the results.

\section{DISCUSSION}

The development of an ideal and effective CR programme is still being explored and current CR mainly consists of contemporary conventional exercises. In fact, current 
CR programmes have been reported to be underdeveloped and limited, reflected by a poor level of involvement with less than $30 \%$ of patients participating in the existing offerings. ${ }^{28}$ As such, there is an unmet need for reforms and the provision of alternative CR programmes to encourage the growth of CR. The exploration of an ideal CR exercise that is most beneficial for CCS patients should be determined.

This trial is the first one to compare the safety, feasibility and benefits of TCCRP and CER in CCS patients. There are several strengths of our trial: first, the proposed research study is unique and the first study about a Bafa Wubu of Tai Chi which is a new Tai Chi school. Second, TCCRP in this study was specifically designed for patients with CCS. Finally, this is the first time for Tai Chi study to develop a comparative training system to match the conventional exercise. Our study will supply scientific evidence for the promotion of Bafa Wubu of Tai Chi at home and abroad.

TCCRP has some features which make it more suitable for CCS patients. First, the intensity of TCCRP is low, and it is much safer for patients with CCS. Second, TCCRP is much easier to be learnt and possesses a simple structure of movements, a reasonable number of postures and fewer practice environment limitations. Third, TCCRP is not limited by location and easy to be carried out. Finally, TCCRP does not need money or any equipment. To sum up, compared with CER, TCCRP is more suitable for CCS patients.

Compared with conventional exercise styles (eg, aerobic, resistance and extensibility exercise), Tai Chi typically involves a mind-body integration practice that combines the coordination of slow movements with mental focus, deep breathing and relaxation for promoting both physical and mental well-being. ${ }^{29-31}$ Previous studies have shown that regular Tai Chi exercise is beneficial in improving psychological and physiological outcomes among the elderly and various clinical populations (eg, Parkinson's disease, diabetes mellitus, hypertension, chronic obstructive pulmonary disease and psychological illness). ${ }^{32-35}$ As a typical mind-body exercise which incorporates the characteristics of TCM, Tai Chi may be considered to be an effective exercise to promote health in a diverse range of populations (eg, healthy population, patients with chronic diseases, youths, middle-aged or elderly adults).$^{36} 37$

Compared with other Tai Chi schools, TCCRP has distinct advantages for CCS patients. TCCRP used Bafa Wubu of Tai Chi, namely, introductory routines to Tai Chi characterised by simple structures. Of the many styles of Tai Chi, however, it is hard to further popularise and generalise, due to its numerous movements and complexity, especially among patients with CCS. By upholding scientific, standardised and simplified principles, the Bafa Wubu of Tai Chi is systematically refined and sorted out on the basis of the other forms of Tai Chi, and the two exercise forms of 'standing' and 'marching', thus forming a set of Tai Chi routines for popularisation characterised by culture, fitness and simplicity. Compared with the others, Bafa Wubu of Tai Chi is safer and much easier to be mastered for patients with CCS.

It should be acknowledged that this study has several limitations. It is difficult to monitor any additional physical activity of participants during the study duration. Although all participants will be required to record their daily physical activity or exercise information with a pedometer, this is not sufficiently accurate to track their daily activity intensity. Furthermore, due to the nature of the exercise interventions (Tai Chi vs CER), the blinding of participants is unachievable in this trial. However, every effort will be made to ensure that the outcome assessors, data managers and statisticians participating in this study will be kept blind of the treatment allocations.

In conclusion, this study aims to assess the efficacy, safety and acceptability of an innovative TCCRP for CCS patients. The finding will be vital to help establish an optimal CR programme for treating CCS patients.

\section{TRIAL STATUS}

This trial is currently in the recruitment phase. Estimated completion of the trial is expected to be completed by December 2020.

\section{Author affiliations}

${ }^{1}$ Department of Cardiology, First Medical Center of Chinese People's Libration Army General Hospital, Beijing, China

${ }^{2}$ College of Physical Education and Sports, Beijing Normal University, Beijing, China ${ }^{3}$ Department of Cardiovascular Medicine, Anzhen Community Health Service Center, Chaoyang District, Beijing, China

${ }^{4}$ Department of Cardiovascular Medicine, Beijing Shuili Hospital, Beijing, China

${ }^{5}$ College of Wushu, Beijing Sport University, Beijing, China

Acknowledgements The authors most gratefully thank the physicians and nurses of the Chinese PLA General Hospital, Beijing Shuili Hospital and Anzhen Community Health Service Center, Chaoyang District, Beijing. Thank you for effort working as numbers of patient public involvement group, such as Weiling Guo, Wu Feng, Haibin Wang, Yong Ma and Jijun Li.

Contributors SjL, JM and $\mathrm{HcY}$ conceived and designed the study protocol. The individual interviews were conducted by SjL, JM, LH, LsZ and AyG. JwZ and JM performed the translation and analysed the data. SjL, JwZ, WY, ZhC, QyW, MzC, YmL, YIL, TmG, ChL, BS and HwW guided and supervised the Tai Chi training. JwZ and JM contributed to writing and reading the manuscript. All authors approved the final manuscript.

Funding This work is financially supported by National Key R\&D Program of China (2018YFC2000600) and Finance Department of the State Administration of Traditional Chinese Medicine (GZY-GCS-2018-011) and the Wushu Research Institute of the General Administration of Sport of China (WSH2018A004).

\section{Competing interests None declared.}

Patient and public involvement We gave priority to patients as far as possible. Patients are the major factor to be consider when designing the trial, subject recruitment and data sharing. Since the very beginning of our study, we have constructed a patient public involvement group. During the group meeting, patient public involvement group members were informed about how the study will be conducted. We always take a lot of time to communicate with patients about how to improve the detail of study to improve their health and they have given some good advice. For example, the patients suggested us to prepare simple booklet to introduce our study during the outpatients' visit and by internet, also to enlarge the recruitment extent by WeChat and app of our hospital. We planned to disseminate our research to the participants and the public, such as publicising our research in hospital official accounts and various academic lectures.

Patient consent for publication Obtained. 
Provenance and peer review Not commissioned; externally peer reviewed.

Open access This is an open access article distributed in accordance with the Creative Commons Attribution Non Commercial (CC BY-NC 4.0) license, which permits others to distribute, remix, adapt, build upon this work non-commercially, and license their derivative works on different terms, provided the original work is properly cited, appropriate credit is given, any changes made indicated, and the use is non-commercial. See: http://creativecommons.org/licenses/by-nc/4.0/.

ORCID iD

Jian wei Zhang http://orcid.org/0000-0002-8301-3047

\section{REFERENCES}

1 Lozano R, Naghavi M, Foreman K, et al. Global and regional mortality from 235 causes of death for 20 age groups in 1990 and 2010: a systematic analysis for the global burden of disease study 2010. Lancet 2012;380:2095-128.

2 Mirzaei M, Truswell AS, Taylor R, et al. Coronary heart disease epidemics: not all the same. Heart 2009;95:740-6.

3 Taylor RS, Brown A, Ebrahim S, et al. Exercise-based rehabilitation for patients with coronary heart disease: systematic review and meta-analysis of randomized controlled trials. Am J Med 2004;116:682-92.

4 Goel K, Lennon RJ, Tilbury RT, et al. Impact of cardiac rehabilitation on mortality and cardiovascular events after percutaneous coronary intervention in the community. Circulation 2011;123:2344-52.

5 Suaya JA, Stason WB, Ades PA, et al. Cardiac rehabilitation and survival in older coronary patients. J Am Coll Cardiol 2009;54:25-33.

6 Smith SC, Benjamin EJ, Bonow RO, et al. AHA/ACCF secondary prevention and risk reduction therapy for patients with coronary and other atherosclerotic vascular disease: 2011 update: a guideline from the American heart association and American College of cardiology Foundation. Circulation 2011;124:2458-73.

7 Levine GN, Bates ER, Blankenship JC, et al. 2011 ACCF/AHA SCAI guideline for percutaneous coronary intervention. A report of the American College of cardiology Foundation/American heart association Task force on practice guidelines and the Society for cardiovascular angiography and interventions. J Am Coll Cardiol 2011;58:e44-122.

8 Piepoli MF, Hoes AW, Agewall S, et al. 2016 European Guidelines on cardiovascular disease prevention in clinical practice: The Sixth Joint Task Force of the European Society of Cardiology and Other Societies on Cardiovascular Disease Prevention in Clinical Practice (constituted by representatives of 10 societies and by invited experts) Developed with the special contribution of the European Association for Cardiovascular Prevention \& Rehabilitation (EACPR). Eur Heart J 2016;37:2315-81.

9 Ritchey MD, Maresh S, McNeely J, et al. Tracking cardiac rehabilitation participation and completion among Medicare beneficiaries to inform the efforts of a national initiative. Circ Cardiovasc Qual Outcomes 2020;13:e005902.

10 Tao T, He T, Wang X, et al. Metabolic profiling analysis of patients with coronary heart disease undergoing Xuefu Zhuyu decoction treatment. Front Pharmacol 2019;10:985.

11 Salmoirago-Blotcher E, Wayne PM, Dunsiger S, et al. Tai chi is a promising exercise option for patients with coronary heart disease declining cardiac rehabilitation. J Am Heart Assoc 2017;6:006603.

12 Taylor-Piliae RE, Silva E, Sheremeta SP. Tai chi as an adjunct physical activity for adults aged 45 years and older enrolled in phase III cardiac rehabilitation. Eur J Cardiovasc Nurs 2012;11:34-43.

13 Sato S, Makita S, Uchida R, et al. Effect of tai chi training on baroreflex sensitivity and heart rate variability in patients with coronary heart disease. Int Heart J 2010;51:238-41.

14 Liu T, Chan AW, Liu YH, et al. Effects of tai Chi-based cardiac rehabilitation on aerobic endurance, psychosocial well-being, and cardiovascular risk reduction among patients with coronary heart disease: a systematic review and meta-analysis. Eur J Cardiovasc Nurs 2018;17:368-83.
15 Zhang J, Lyu S, Wang J, et al. A comparative study on the therapeutic effect of Taijiquan on patients with type 2 diabetes mellitus of different genders. J Beijing Normal University 2019;55:545-50.

16 Lyu S. Bafa Wubu of tai chi. Beijing: Beijing Sport University Press, 2018: 18-20.

17 Knuuti J, Wijns W, Saraste A, et al. Esc guidelines for the diagnosis and management of chronic coronary syndromes: the task force for the diagnosis and management of chronic coronary syndromes of the European Society of cardiology (ESC). Eur Heart $J$ 2020;41:407-77.

18 Salvetti XM, Oliveira JA, Servantes DM, et al. How much do the benefits cost? effects of a home-based training programme on cardiovascular fitness, quality of life, programme cost and adherence for patients with coronary disease. Clin Rehabil 2008;22:987-96.

$19 \mathrm{Xu}$ Z. Study on the perioperative nursing practice of TCM auricular compression combined with mindfulness meditation training in patients with colorectal cancer. J Liaoning Univ Tradit Chin Med 2020;22:218-20.

20 Borg GA. Psychophysical bases of perceived exertion. Med Sci Sports Exerc 1982;14:377???381-81.

21 Fletcher GF, Ades PA, Kligfield P, et al. Exercise standards for testing and training: a scientific statement from the American heart association. Circulation 2013;128:873-934.

22 Piepoli MF, Conraads V, Corrà U, et al. Exercise training in heart failure: from theory to practice. A consensus document of the heart failure association and the European association for cardiovascular prevention and rehabilitation. Eur J Heart Fail 2011;13:347-57.

23 Ware JE, Gandek B. Overview of the SF-36 health survey and the International quality of life assessment (IQOLA) project. J Clin Epidemiol 1998;51:903-12.

24 Yang T. Psychological stress of urban population in social transition. Chin J Epidemiol 2002;6:64-6.

25 You Y, Wen A. Human balance assessment method. Chin J Rehabil Med 2014;29:1099-104.

26 Berg K, Wood-Dauphinee S, Williams Jl. The balance scale: reliability assessment with elderly residents and patients with an acute stroke. Scand J Rehabil Med 1995;27:27-36.

27 Buysse DJ, Reynolds CF, Monk TH, et al. The Pittsburgh sleep quality index: a new instrument for psychiatric practice and research. Psychiatry Res 1989;28:193-213.

28 De Gruyter E, Ford G, Stavreski B. Economic and Social Impact of Increasing Uptake of Cardiac Rehabilitation Services--A Cost Benefit Analysis. Heart Lung Circ 2016;25:175-83.

29 Nery RM, Zanini M, Ferrari JN, et al. Tai chi Chuan for cardiac rehabilitation in patients with coronary arterial disease. Arq Bras Cardiol 2014;102:588-92.

30 Yang $\mathrm{Y}-\mathrm{L}$, Wang $\mathrm{Y}-\mathrm{H}$, Wang S-R, et al. The effect of tai chi on cardiorespiratory fitness for coronary disease rehabilitation: a systematic review and meta-analysis. Front Physiol 2017;8:1091.

31 Lan C, Chen SY, Lai JS, et al. The effect of tai chi on cardiorespiratory function in patients with coronary artery bypass surgery. Med Sci Sports Exerc 1999;31:634-8.

32 Chang R-Y, Koo M, Yu Z-R, et al. The effect of t'ai chi exercise on autonomic nervous function of patients with coronary artery disease. $J$ Altern Complement Med 2008;14:1107-13.

33 Gao Q, Leung A, Yang Y, et al. Effects of tai chi on balance and fall prevention in Parkinson's disease: a randomized controlled trial. Clin Rehabil 2014;28:748-53.

34 Xiao C-M, Zhuang Y-C. Effects of tai chi ball on balance and physical function in older adults with type 2 diabetes mellitus. J Am Geriatr Soc 2015;63:176-7.

35 Chan AWK, Lee A, Lee DTF, et al. The sustaining effects of tai chi Qigong on physiological health for COPD patients: a randomized controlled trial. Complement Ther Med 2013;21:585-94.

36 Chen WW, Sun WY. Tai chi chuan, an alternative form of exercise for health promotion and disease prevention for older adults in the community. Int Q Community Health Educ 1996;16:333-9.

37 Guo J-B, Chen B-L, Lu Y-M, et al. Tai chi for improving cardiopulmonary function and quality of life in patients with chronic obstructive pulmonary disease: a systematic review and metaanalysis. Clin Rehabil 2016;30:750-64. 